\title{
A New Game for Future Wi-Fi Spectrum Sharing
}

\author{
Tomas Cuzanauskas ${ }^{1}$, Yoram Haddad ${ }^{2}$ \\ ${ }^{1}$ Department of Telecommunication Engineering, Vilnius Gediminas Technical University, \\ Naugarduko 41, LT-03227 Vilnius, Lithuania \\ ${ }^{2}$ Department of Computer Science, Jerusalem College of Technology, \\ Givat Mordehai 21, Havaad Haleumi St. 93721 Jerusalem, Israel \\ tomas.cuzanauskas@vgtu.lt
}

\begin{abstract}
This article offers a vision for moving on from the paradigm of "constrained politeness" that has long governed Wi-Fi access to a shared spectrum - as embodied by the CSMA/CA mechanism and restricted EIRP limits. Instead, it is proposed to embrace a paradigm of a "cooperative game" where quasi-cognitive devices establish by themselves a mutually collaborative spectrum coexistence community, based on a unified set of simple rules, which operates on the basis of balancing individual transmits powers as a function of overall noise environment. It is shown that the proposed new mode of spectrum access, results in a significantly increased overall spectrum use efficiency, defined in terms of aggregate throughput carried in given bandwidth by a populace of devices over unit area. Most importantly, the new spectrum access would be still implicitly suitable for the Wi-Fi ethos of self-management and uncoordinated deployment.

The proposed method, supported by both theoretical and practical simulations, represents one of the first practical implementations that prove feasibility and usefulness of Game Theory to the practice of wireless communications.
\end{abstract}

Index Terms - Cognitive radio; communications technology; IP networks; radio spectrum management; software defined networking; wireless networks.

\section{INTRODUCTION}

Seen from the perspective of a radio spectrum manager, Wi-Fi embodies both what is the best and what is the worst about the "commons" - frequency bands that allow unlicensed shared access such as the $2.4 \mathrm{GHz}$ ISM band. On the positive side, the freedom of unlicensed access guarantees easy market entry for any type of wireless devices, which allows building a rich eco-system of wireless innovation. Yet, such a system becomes a victim of its own success as growing density of devices coupled with unmanaged spectrum access gives a rise in frequency of clashes of transmissions from different devices and results in interference and deterioration of quality and throughput of wireless links.

By being aware of these risks of overexploitation, spectrum managers try preserving a modicum of control over commons by setting some general constraints on radios using those bands - most typically maximum transmit

Manuscript received 30 September, 2016; accepted 11 January, 2017.

The concept of New Spectrum Sharing Wi-Fi Rules was developed as a group activity within COST Action IC0905 TERRA (www.cost-terra.org). power, but also other parameters such as Duty Cycle or maximum transmitter ON-time. For instance, in Europe Wi$\mathrm{Fi}$ in $2.4 \mathrm{GHz}$ band is only allowed to transmit with maximum Effective Isotropically Radiated Power (EIRP) of $100 \mathrm{~mW}$. Similar rules are applied for $5 \mathrm{GHz}$ band as well. This constitutes a serious inhibitor that dramatically limits the communication range and link quality as well as the effective throughput of wireless applications. For instance, brief calculations with the $100 \mathrm{~mW}$ max. EIRP (mentioned above) leads us to a typical range of about $150 \mathrm{~m}$, if we consider a receiver sensitivity of $-70 \mathrm{dBm}$ and an optimistic Free Space Loss model. It will likely be even worse if we consider tough indoor fading conditions. Such a paradigm for constructing unlicensed spectrum access rules is now decades old and this article describes a novel way of sharing unlicensed bands with more efficiency with less limitation for deployment of innovative systems with higher bandwidth and service quality.

The next section will provide a closer look at problems with radio channel access in current $\mathrm{Wi}-\mathrm{Fi}$ solutions. It is followed by the description in third section of the proposed alternative Game Theoretic (GT) radio spectrum access model, supported by modeling results. This would be complemented by fourth section with case studies of impact on incumbent Wi-Fi spectrum access technologies, before offering final conclusions.

\section{State-OF-THE-ART IN WI-FI RAdio ChanNEL ACCESS}

Since the beginning, the IEEE 802.11 standard had established two mechanisms that govern access to radio channel, namely: DCF (Distributed Coordination Function) and PCF (Point Coordination Function). The DCF mechanism is the only one that has been certified by the WiFi Alliance and as of today, it is the access mode of choice implemented in all the Wi-Fi compatible devices. DCF works by employing a mechanism called CSMA/CA (Carrier Sensing Multiple Access/Collision Avoidance). The essence of CSMA/CA is that a Wi-Fi transceiver senses the channel before transmitting a packet, and if upon transmission it detects that collision did occur (i.e. when two transceivers tried transmitting at the same time), then the transceiver waits for some time before trying to re-send the packet [1]. All stations, which participate for wireless medium access, perform clear channel assessment (CCA). 
This is done by using both virtual carrier sense mechanism as well as physical (transmit power) energy detection mechanism. The period for which the CCA is performed is called DCF inter-frame space (DIFS). If the medium is idle for such period, stations can occupy channel and start transmissions. If during CCA period wireless medium is busy, stations wait for another DIFS plus a random back off period. Upon gaining wireless medium station, it maintains the occupation of channel by using short inter-frame space (SIFS) between data packets. Therefore, none of other stations can interrupt the transmission since they are sensing channel for DIFS period, which is longer compared to SIFS. However, IEEE 802.11 has transmission time rules, which prevents individual station from occupying the wireless medium for too long.

As mentioned before, CSMA/CA employs two mechanisms of carrier sense. Energy based detector, which resides in PHY layer and detects energy levels in channel, as well as preambles with frame length. Virtual carrier sense resides in MAC. In basic principle, virtual carrier sense derives packet duration field of the MAC header and takes that duration as a period for which wireless medium will be busy. The virtual carrier sense mechanism is known as Network Allocation Vector (NAV). The medium is considered as idle only when NAV and PHY carrier sense mechanisms indicates that wireless medium is idle. This simple mechanism allows fully decentralized and uncoordinated operation of transceivers and therefore was favored by the manufacturers. However, this mechanism exhibits poor performance in highly interfered environments. Despite its drawbacks DCF received an update, Enhanced Distributed Channel Access, which adds Quality of service capabilities, however uses the same wireless medium access approach as DCF.

The exploding number of Wi-Fi devices in $2.4 \mathrm{GHz}$ band, all typically operating within only 3 non-overlapping channels, generates such a high amount of interference in dense urban environments that the using of PCF has been recently re-considered. This would require to use a central coordinating unit - an Access Point (AP), as a "master" of the cluster of devices. Alternatively, some recent works had proposed schemes for self-governed appointment of master in the distributed environments without single AP, where the point coordinator is chosen among all the participants [2]. But despite that, the use of PCF requires coordination among devices, which creates an additional level of complexity and stifles the dynamism of Wi-Fi deployment and operation.

Although we mentioned above that there are only 3 nonoverlapping channels, it worth to mention that recent efforts show that the introduction of a $4^{\text {th }}$ channel can lead to a total of four partially overlapping channels, which exhibit minimal degradation in the QoS of VoIP traffic and is generally the more sensitive to the signal degradation [3], [4].

Despite the drawbacks, the current Wi-Fi channel access mechanisms have many useful features. Wi-Fi uses adaptive modulation and coding scheme that makes its packet reception highly tolerant of noise and link gain fluctuations, allowing packets to be demodulated at low signal to noise ratios. Reception is also tolerant of frequency offset and receiver oscillator error. This supports Wi-Fi's asynchronous burst modulation operation and allows interfering and desired packets to be received and examined for their source and destination addresses, received signal level, channel of transmission, size, length, inter-arrival time, and modulation rate, amongst other metrics. All this make them highly suitable for employing some more rational channel access method such as that proposed in this article.

Wi-Fi devices often possess powerful (mostly proprietary) channel sensing capabilities that support carrier sensing in the CSMA/CA protocol. In essence, many of the existing attributes, commonly embodied within Wi-Fi will support radio cognition if they are made available to higher layer coexistence/collaboration processes. A growing number of Wi-Fi devices already have embedded cognitive functions implemented as proprietary solutions, e.g. [5], aiming to enhance Wi-Fi availability, data throughput and Quality of Service (QoS), by undertaking dynamic frequency access, radiation direction selection, and dynamic power control. However, as promising as the potentially new Cognitive Radio (CR) attributes of Wi-Fi are the greatest obstacle to $\mathrm{Wi}-\mathrm{Fi}$ performance and IEEE 802.11 evolution remains in the use of the CSMA/CA protocol. This situation is further exacerbated by the Media Access Control (MAC) system becoming encumbered with excessive inter-device signaling, mostly constituting beacons, association/de-association, authentication and probe management messages related to maintaining link integrity and security, which constitute the bulk of Wi-Fi channel occupancy [6]. A significant amount of bandwidth is also used to support the retransmission that grows exponentially with congestion. Over the time that the Wi-Fi standard has been in place, many attempts have been made to manipulate the CSMA/CA protocol in a variety of ways, such as adjusting contention window sizes [7], embedding adaptive threshold channel sensing or instituting changes to the IEEE 802.11 standard, in order to better utilize the use of Request-To-Send/Clear-To-Send (RTS/CTS) messaging. However, the benefits of improved RTS/CTS and similar changes remained questionable, whilst proprietary changes to the protocols (outside the IEEE 802.11 standard) can further deteriorate performance [8].

\section{A NEW GAME FOR SHARED SPECTRUM USE}

A number of GT algorithms and protocols have been proposed for optimizing channel assignment and power allocation [9], [10]. The essence of GT modeling is that each device needs to be considered a selfish player and their interactions are strategic in a sense that a given players' payoff depends on the other players' actions. This is very fitting for the context of unlicensed Wi-Fi operation in common bands, where devices compete for the airtime, leading to an opportunistic behavior and at some point, spectrum congestion. It is a dynamic environment, difficult to analyze and for which it is difficult to provide sound resource management schemes. Standard analytical models no longer cope with the increasing complexity and dynamics of such eclectic communications environment, whereas GT provides a framework in which the paradigm shifts to a more flexible and efficient resource sharing that might 
eventually materialize.

Within the GT framework, the key problem is to design distributed resource allocation rules, which would lead to a Nash equilibrium that is efficient and possibly fair [9], [10]. These rules would be self-enforcing and therefore would not require an external intervention to verify its compliance.

Note that the channel access framework proposed here may be described as a non-cooperative game, meaning that the decisions are taken autonomously by the devices (i.e. no coalition is required for decision making purposes). Yet, non-cooperative does not mean non-collaborative; a certain amount of communication among the devices may be assumed (there are games for which this may not be necessary; ideally, if the sensing and context awareness are perfect, signaling would be minimal).

In the proposed game, the power allocation is determined as the outcome, hence, representing a distributed, interference-aware, power allocation game. In a wireless network of $\mathrm{N}$ independent transmit-receive pairs $\left(\mathrm{Tx}_{\mathrm{i}}-\mathrm{Rx}_{\mathrm{i}}\right)$, each pair would be considered a player in the game. The objective of the game is to find stable points of power allocation for each player so that the players' global utility is maximized while the cumulated power levels are kept to a minimum

When taking into consideration the composition of players, it is also important to consider master-slave relationship aspect. In the context of $\mathrm{Wi}-\mathrm{Fi}$ operation, the game models a set of primary master devices - Agents, such as APs, each having one or more slaves, or Station Terminals, see Fig. 1.

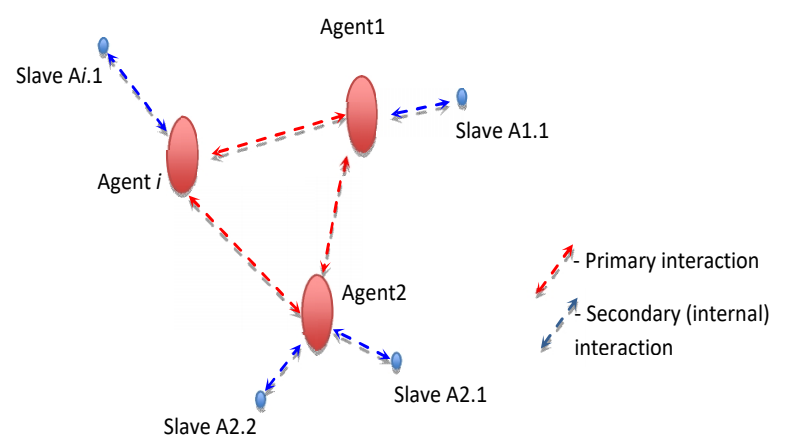

Fig. 1. Depiction of Power Coordination Game players.

The Agents are competing between each-other for a share of spectrum throughput. The slaves are implicitly appearing as a secondary tier and do not need to be part of the game strategy. In the case when AP has more than one slave, it could use its own scheduling to partition available throughput between its served slaves, which is in line with the current Wi-Fi deployment construct under Infrastructure Mode operation and complies with the users' expectations. If collaboration for the sake of improved efficiency was deemed beneficial, the Agents might exchange information over communications links established either over the air, or by means of wire line TCP/IP links. This would be consistent with the Wi-Fi networking architecture. While currently Wi-Fi APs do not normally communicate with each other by means of their wire line connections, the raising of Software Defined Network paradigm, this collaboration could now be more realistic [11], [12]. For instance [13] a realistic use case where SDN can be leveraged at the Base Station level to improve collaboration and interference reduction. Each AP can update its status to a controller via South-Bound Interface (SBI). Then the controller can perform some optimization routine that has been developed in a dedicated Application on the top of the control plane via the North Bound Interface (NBI) [12]. This is especially important when handling multiple APs deployments at infrastructural network topology, which requires frequency re-use [2]. The frequency re-use requires additional protocol changes as well as efficient power management between APs and CPEs, in order to minimize the effects of self-interference when operating on the same tower by re-using small chunk of spectrum allocated to specific WISP; see Fig. 3 below of such a deployment.

In Fig. 2 we show how SDN enable cooperation between two APs to enable seamless roaming. Since the controller know ahead of time that a user is moving toward edge of the cell (monitored by RSSI etc.), it can trigger the roaming so that no disconnection is experienced. In the same way, the physical signal level information can be used for the channel allocation.

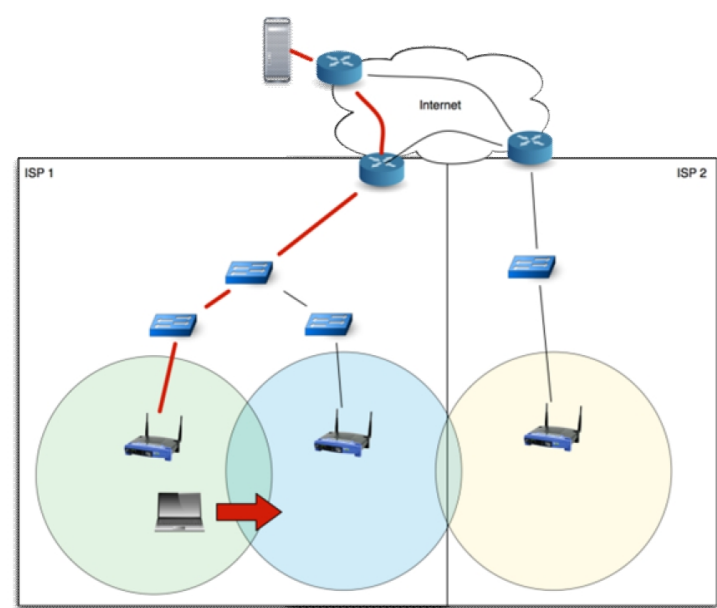

Fig. 2. APs collaboration with SDN framework to enable seamless roaming.

The game only needs to simulate a sub-set of finite size from a total populace of Agents that might be located in a given area, i.e. those devices that may be considered forming "coexistence community". The coexistence community would be made of Agents that share a common part of spectrum, i.e. a given channel. It may also change with time, if the channel is changing as a result of using Dynamic Frequency Selection mechanism or otherwise.

In Fig. 3 we have a typical frequency re-use deployment, where different APs are sharing same frequency. In example, A, APs are using $5500 \mathrm{MHz}$ frequency and in $\mathrm{B}$, APs are re-using $5520 \mathrm{MHz}$ frequency with possible channel width of $20 \mathrm{MHz}$. As it can be noticed, the important part here is the $\mathrm{Tx}, \mathrm{Rx}$ synchronization between the group A APs and the group B Aps. This is achieved by implemented TDMA based protocol, which handles the synchronous transmission, however, in such a deployment, the transmit power from CPEs: $\mathrm{C} 1, \mathrm{C} 2, \mathrm{C} 3, \mathrm{C} 4$ plays an important role, by minimizing the effects of far end and near end interference. It is known that antennas can still hear some part of the noise coming from CPE C1 to AP, which transmits to $\mathrm{CPE} \mathrm{C2}$; therefore, it is important to use power 
control allocation between clients of far and near end. This is an important part of our proposed game theory model.

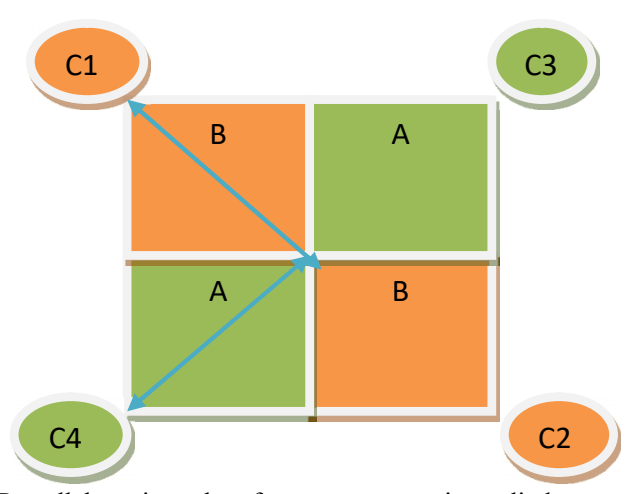

Fig. 3. APs collaboration when frequency re-use is applied.

The proposed power coordination game was built and tested with the following key objectives in mind:

- To ensure self-governed access to the shared (ISM) bands with the objective of improving the global spectralspatial efficiency of the band, e.g. measured as the total aggregate traffic of all links in given a locality/area;

- To ensure that the newly deployed devices built on the GT operating paradigm do not create excessive interference and disruptions to the legacy CSMA/CAbased devices. In fact, the latter devices might still participate passively in the channel access game to a certain extent as players with fixed strategies. There will though remain a risk that the legacy devices might become victims of "exposed node" phenomenon and be effectively prohibited from attempting transmission. This would be considered in the fourth section.

The expanded mathematical apparatus to describe the working of the game was offered in [14], [15]. A simple set of rules that could be easily handled by each and every participating Agent on their own:

- Agents initiate their work with a default EIRP of 100 $\mathrm{mW}$;

- Each Agent will have randomly generated internal target throughput, in order to model the realistic fluctuation of data traffic, because not every Wi-Fi terminal will need to transmit at maximum bit rate at any given time;

- Each Agent will set-up links with its secondary slaves and will proceed exchanging information using random channel access: when you have data to send, send it, if data does not come through, re-send it later;

- At randomized time instances, each Agent evaluates its Signal to Interference plus Noise Ratio (SINR) and if it has changed since previous check, calculates the new transmit power, which is inversely proportional to actual SINR (for details and formulae the reader is referred to [15]). Then:

- by using adjusted Shannon formula of communications, link throughput as a function of noise, the Agent checks whether newly calculated transmit power will allow meeting its instantaneous target throughput:

- if the achievable throughput is equal or less than the target, then Agent sets the new power level and returns to a normal operating mode as per step 3;

- if the achievable throughput exceeds the target, the target power will be downscaled to just meet the target throughput, before returning to a normal operating mode as per step 3;

- Return to repeat steps 3-4 until the system reaches equilibrium. In real life implementation, this would mean that all Agents cycle between steps 3-4 indefinitely while in operation.

In essence, this means that instead of having any carrier sensing or timing considerations with regard to access the radio channel, the $\mathrm{Wi}-\mathrm{Fi}$ Agents will constantly be in the state of optimally balanced transmit power versus background noise, which should ensure the instantaneous transmission of the message using random channel access. This means that Wi-Fi devices could also be transmitting incessantly, which was actually how the process was simulated when establishing total achievable aggregated data throughputs.

Note that under the proposed power coordination game, the active collaboration between agents is, strictly speaking, not necessary as a departing point, and the simulations reported herein, were done with agents acting without communicating with each other. This brings forth the analogy of the modeled ,coexistence community” of Wi-Fi devices with biological systems (bees, termites), because in both systems individual agents achieve common purpose such as maximizing the sum of pay-offs (for the social benefit of the community) and where everyone employs a set of internalized simple and identical rules. So by way of following the common set of algorithmic rules, the agents realize implicit social coordination and collaboration functionality. However, having means of communication between Agents - APs (i.e. via control channel, or consulting a centralized database or each other via wired $\mathrm{TCP} / \mathrm{IP}$ connectivity) might be useful future addition, as it would allow a possibility of building distributed sensing strategies, akin to chemical markers used by the agents in the biological systems.

By using the tool [16] a set of theoretical simulations had been performed by using ITU indoor propagation model P.1238 and assuming co-channel operation [15]. The number of links, each representing one user, randomly distributed in an area of 100x100 m, varied between 2 and 10 and the main result was expressed in terms of aggregate channel-area capacity, i.e. sum of capacities of all deployed individual links. The simulations show that the proposed power coordination game allows achieving significantly larger aggregate capacity of Wi-Fi links in a given bandwidth compared with the baseline case of current deployment with $100 \mathrm{~mW}$ EIRP limit (Fig. 4).

The simulation results are especially striking considering the rather small scale of the simulated scenario, where higher power would be normally not considered necessary thanks to favorable link budget over such short distances. This illustrates that by removing the artificial EIRP limit, it allows Wi-Fi devices to tap into additional power margin when needing to improve SINR and subsequently link quality. At the same time, these results prove that given the clear and simple power game rules, the system would converge to some equilibrium state and no excessive overexploitation of common band would occur.

Additionally, after modification of previous tool, a Point 
to Multi-Point Scenario was simulated. In this case a group of 8 Master nodes with 4 connected Slave nodes per each Master node was used. By removing EIRP limit, allocated devices are able to reach the highest system capacity, which proves that simple power rules can be applied in such system without overall system degradation (Fig. 5).

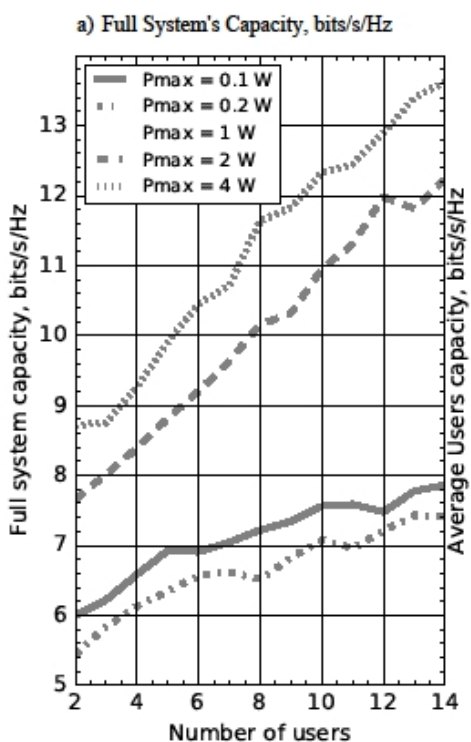

b) Average Users Capacity, bits/s/Hz

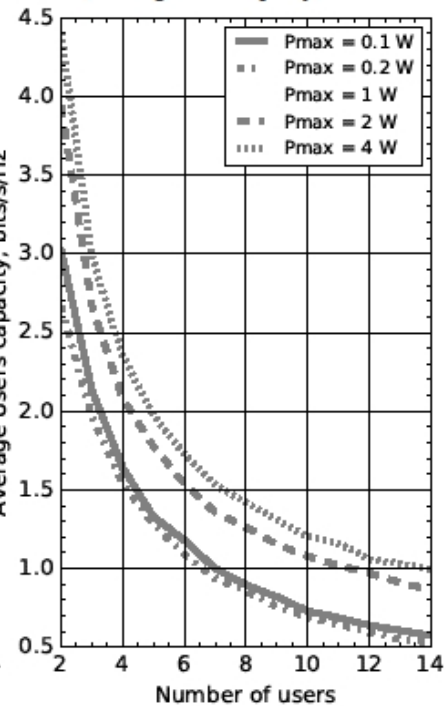

Fig. 4. Aggregate channel-area capacity for Wi-Fi devices employing interference-aware power coordination game $(100 \times 100 \mathrm{~m}$ area) a) Full Systems Capacity b) Average Users Capacity.
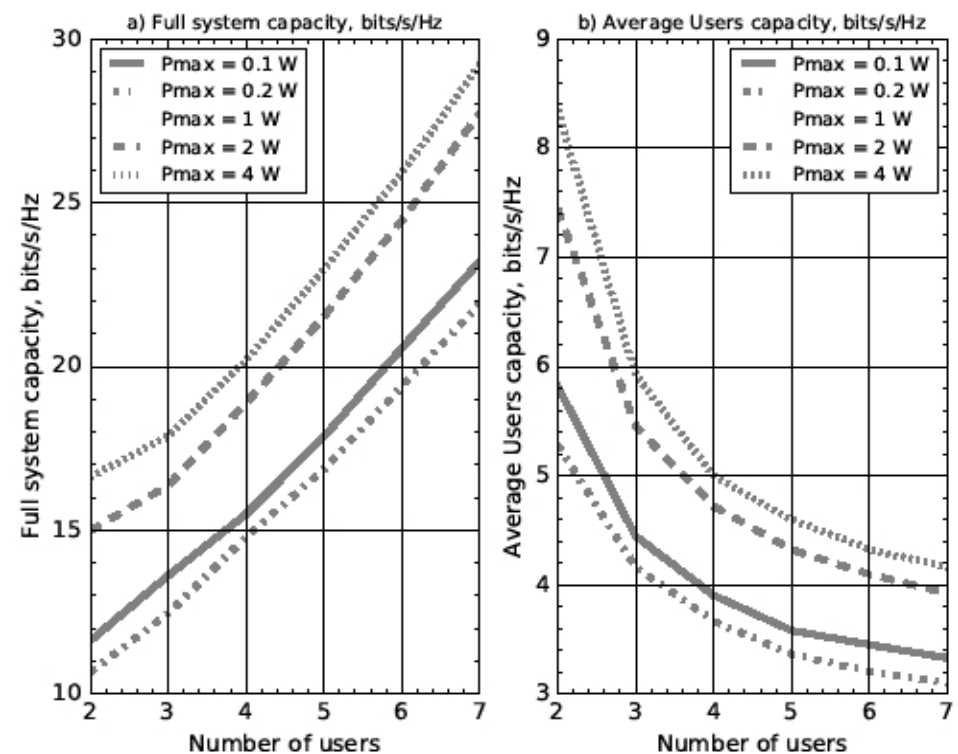

Fig. 5. Aggregate channel-area capacity in PtMP for Wi-Fi devices employing interference-aware power coordination game $(100 \times 100 \mathrm{~m}$ area) a) Full Systems Capacity b) Average Users Capacity.

Finally, outdoor based experiments were performed by employing IEEE 802.11ac hardware and adding necessary modification. During testing, it was monitored how the environmental impact of third party operating APs changed the performance on proposed protocol. Outdoor protocol is based on multi-polling based protocol with similar operation as HCCA mechanism proposed by IEEE 802.11e [17] using Point-to-Multipoint (PtMP) scenario with off-the-shelf IEEE 802.11 ac devices. These include total of 5 CPEs devices at $0.5 \mathrm{~km}$ and $200 \mathrm{~m}$ distances. The AP is connected with 90 degree sector antenna, which has gain of $20 \mathrm{dBi}$. The signal, depending on the distance, was in the range from $-45 \mathrm{dBm}$ up to $-50 \mathrm{dBm}$. The tests were performed in a variable radio environment. The output power was self-stabilizing depending on Signal-to-Noise-Ratio (SNR) requirements. Performance test of the operating links was done using iPerf tool and results are shown in Figure 6.

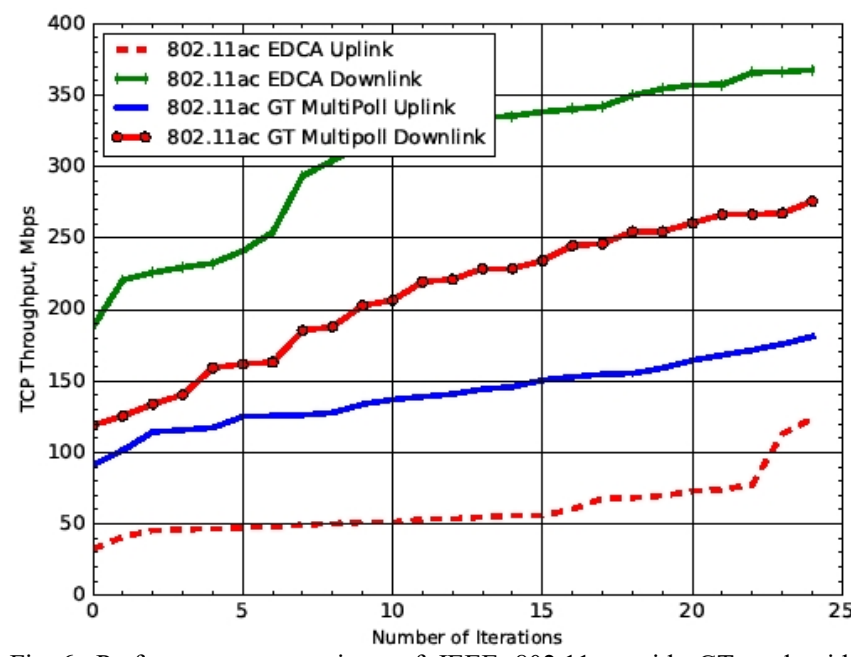

Fig. 6. Performance comparison of IEEE 802.11ac with GT and with standard EDCA wireless medium access mechanisms. 
In PtMP scenario, the throughput of $802.11 \mathrm{ac}$ CSMA/CA links decreased due to self-collisions from clients connected to the same Ap. This is typical hidden node problem on current IEEE 802.11ac devices, which causes high back-off times and decreases the total AP capacity.

\section{COEXISTEnCE OF "NeW" Vs. "OLD” DEVICES}

Whenever considering such a fundamental operational platform shift, as described in this article, it is obvious to ponder how the appearance of "new" devices would impact the operation of the existing large populace of "old" devices. Therefore, additional set of simulations was carried out using the same software tool [16], with the aim to consider the effect of varying proportions of devices with GTenabled power control function on the incumbent Wi-Fi devices, which are constrained by the use of CSMA/CA and EIRP ceiling (of $100 \mathrm{~mW}$ ).

To establish a reference base, the clean slate scenario was first simulated, where all devices would be either powerrestricted or GT-enabled users. For this comparative simulation, a new metric was used, by expressing the results in terms of "user satisfaction" of Wi-Fi devices, i.e. the percentage of users who achieved their throughput requirements. The results obtained for homogeneous clusters that consist of solely one type of devices are shown in Fig. 7.

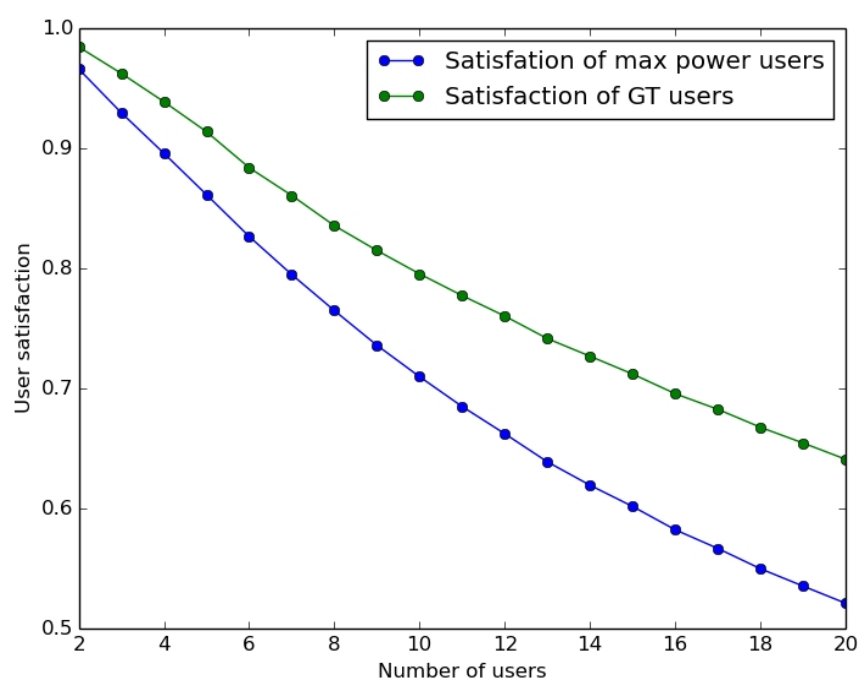

Fig. 7. Achieved individual data throughput objectives: homogeneous device cluster in $100 \times 100 \mathrm{~m}$ area.

The simulated results shown in Fig. 7 illustrate the well understood (and already observed in real life) tendency that the growing number of closely co-located users reduces the effective throughput available to individual link. However, the GT-enabled users on average show some $20 \%$ higher satisfaction rates.

The following simulation considered mixed scenario with varying proportion of GT-enabled versus legacy Wi-Fi devices. The results are shown in Fig. 8.

The results show that by reducing the proportion of incumbent $100 \mathrm{~mW}$ devices, due to proliferation of more power agile GT-enabled devices, it leads up to $20 \%$ for "last man standing" proportional decline of link availability for their users. However, this would be offset by the growth of total link availability for the larger community of devices.

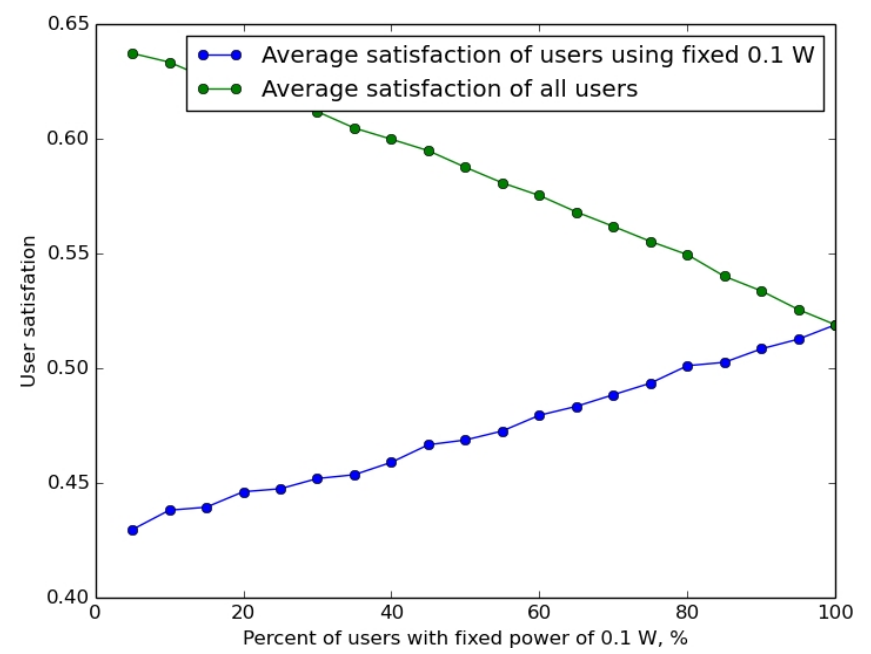

Fig. 8. Achieved individual data throughput objectives: mixed device cluster in $100 \times 100 \mathrm{~m}$ area.

Most importantly, the suffering of incumbent devices was shown to be minor; far from pessimistic scenario of total shutdown due to over-dominance of much more powerful GT-enabled devices.

\section{CONCLUSIONS}

This article projected a future where $\mathrm{Wi}-\mathrm{Fi}$ and other wireless devices, using ISM bands, would form a fluid spectrum sharing communities, with fair and fast channel access ensured by constant balance of power versus environmental noise. It was shown that this equilibrium state may be achieved if quasi-cognitive Wi-Fi devices, which use Game Theoretic algorithm for power coordination and are based on each device following the same set of simple rules. The proposed algorithm was tested in both, theoretical simulations and practical experiments with several Wi-Fi nodes in indoor environment. These studies confirmed that the proposed algorithm allows significant increase of the total capacity of co-located co-channel links in ISM bands. Moreover, it was proven that gradual proliferation of new devices would not cause undue constraints on using older incumbent equipment based that uses current Wi-Fi channel access mechanisms and low EIRP limitations.

The practical experiments proved that the algorithm allows achieving reasonable stability of transmit power and fairness of channel access between neighbouring devices. This suggests that the proposed intelligent interferenceaware power control mechanism may be used as an improvement for CSMA/CA, which shows growing inadequacy and inefficiency due to exploding density of WiFi devices and wasteful signalling overhead.

\section{REFERENCES}

[1] Eldad Perahia, Robert Stacey, Next generation wireless LANS $802.11 \mathrm{n}$ and 802.11ac. New York: Cambridge University Press, pp. 228-230, 2013. [Online]. Available: http://doi.org/10.1017/ CBO9781139061407

[2] Z. Yang, J. Zhang, K. Tan, Q. Zhang, Y. Zhang, "Enabling TDMA for today's wireless LANs", IEEE INFOCOM, 2015, pp. 1436-1444. [Online]. Available: http://dx.doi.org/10.1109/INFOCOM.2015. 7218521

[3] R. Chavez-Santiago, Y. Haddad, V. Lyandres, I. Balasingham, "VoIP transmission in Wi-Fi networks with partially-overlapped channels", in Proc. IEEE Wireless Communications and Networking Conf. (WCNC), 2015. [Online]. Available: http://dx.doi.org/10.1109 /WCNC.2015.7127493 
[4] R. Chavez-Santiago, M. Szydelko, A. Kliks, F. Foukalas, Y. Haddad, K. Nolan, M. Kelly, M. Masonta, I. Balasingham, "5G: the convergence of wireless communications", Journal of Wireless Personal Communications, vol. 83, no. 3, pp. 1617-1642, 2015 [Online]. Available: http://dx.doi.org/10.1007/s11277-015-2467-2

[5] White Paper: "Getting the Best Wi-Fi Performance Possible through Both Beamforming and Adaptive Antennas", 2013 Ruckus Networks. [Online]. Available: http://c541678.r78.cf2.rackcdn.com/wp/wpusing-all-the-tools-you-can.pdf

[6] S. Hanna, J. Sydor, "Distributed sensing of Spectrum Occupancy and Interference in Outdoor $2.4 \mathrm{GHz} \mathrm{Wi}-\mathrm{Fi}$ networks", in Proc. IEEE Global Communications Conf., 2012, pp. 3-7. [Online]. Available: http://dx.doi.org/10.1109/GLOCOM.2012.6503318

[7] J. Zhang, H. Shen, K. Tan, R. Chandr, Y. Zhang, Q. Zhang, "Frame retransmissions considered harmful: improving spectrum efficiency using micro-ACKs", in Proc. Mobicom, 2012, pp. 22-26. [Online]. Available: http://dx.doi.org/10.1145/2348543.2348557

[8] I. Tinnirello, D. Giustiniano, L. Scalia, G. Bianchi, "On the sideeffects of proprietary solutions for fading and interference mitigation in IEEE 802.11b/g outdoor links", Computer Networks, vol. 53, no. 2 , pp. 141-152, 2009. [Online]. Available: http://dx.doi.org/10.1016/j.comnet.2008.10.006

[9] R. Etkin, A. Parekh, D. Tse, "Spectrum sharing for unlicensed bands", IEEE J. Select. Areas Comm., vol. 25, pp. 517-528, 2007. [Online]. Available: http://dx.doi.org/10.1109/JSAC.2007.070402

[10] D. Kreutz, et al., "Software-defined networking: A comprehensive survey", in Proc. IEEE, 2015. [Online]. Available: http://dx.doi.org/10.1109/JPROC.2014.2371999

[11] I. T. Haque, N. Abu-Ghazaleh, "Wireless software defined networking: a survey and taxonomy", in IEEE Communications Surveys \& Tutorials, vol. 18, no. 4, pp. 2713-2737, 2016. [Online]. Available: http://dx.doi.org/10.1109/COMST.2016.2571118

[12] C. Chaudet, Y. Haddad, "Wireless software defined networking", Software Defined Mobile Networks (SDMN): Beyond LTE Network Architecture, pp. 45-60, 2015. [Online]. Available: http://dx.doi.org/ 10.1002/9781118900253.ch4

[13] D. Bercovich, L. M. Contreras-Murillo, Y. Haddad, A. Adam, C. J. Bernardos, "Software-defined wireless transport networks for flexible mobile backhaul in 5G systems", Journal of Mobile Networks and Applications, vol. 20, no. 6, pp. 793-801, 2015. [Online]. Available: http://dx.doi.org/10.1007/s1 1036-015-0635-y

[14] B. Wang, Y. Wu, K. J. R. Liu, "Game theory for cognitive radio networks: an overview", The Int. J. of Computer and Telecommunications Networking, vol. 54, no. 14, pp. 2537-2561, 2010. [Online]. Available: http://dx.doi.org/10.1016/j.comnet. 2010.04 .004

[15] A. Medeisis, J. Sydor, L. Cremene, O. Holland, A. Anskaitis, D. Wiecek, Y. Haddad, T. Cuzanauskas, "ISM-advanced: improved access rules for unlicensed spectrum", in Proc. 7th IEEE Symposium on New Frontiers in Dynamic Spectrum Access Networks (DySPAN 2014), McLean, USA, 2014, pp. 1-4. [Online]. Available: http://dx.doi.org/10.1109/DySPAN.2014.6817796

[16] Software program used for simulation of Power Coordination Game. [Online]. Available: http://www.cost-terra.org/our-publications

[17] T. Cuzanauskas, A. Anskaitis. "Innovative wireless medium control algorithm for wireless devices in ISM bands", International Journal of Computer Applications, pp. 1-5, 2016. [Online]. Available: http://dx.doi.org/10.5120/ijca2016910780 